sung 1981 unterdrückten Volkes. Von besonderem Interesse sind die Abschnitte 'Kosova als autonome Provinz des kommunistischen Jugoslawien', 'Serbische Apartheid in Kosova 1989 bis heute' sowie 'Juristische Argumentation im Sinne eines groß-serbischen Rassismus heute'.

Die Geschichte Kosovas innerhalb Jugoslawiens ist in der Hauptsache die ihrer Verfassungen. So analysiert und vergleicht der Autor die vier Verfassungen von 1946, 1953, 1963 und 1974 und erklärt anhand von ausgewählten Artikeln die jeweilige politische und rechtliche Stellung innerhalb des kommunistischen Jugoslawiens und Serbiens. Die letzte Verfassung unter Tito räumte der autonomen Provinz Kosova 'große Rechte' ein (eigene Sprache, eigenes Bildungssystem, Verwaltung..., s. S. 36/37); 1990 hob die serbische Regierung verfassungswidrig diese Autonomie auf, Kosova wurde zum Ghetto, ethnisch gesäubert (s. S. 42 ff.): Schulen, Krankenhäuser, die Universität wurden geschlossen, Serben bekamen die Arbeitsplätze und die Wohnungen der vertriebenen Albaner, Gewalt gegen Frauen und Kinder, Wehrdienstverweigerer...

Ackermann betont die friedliche Gesinnung der Albaner in Kosova, ihr Streben nach dem früheren Autonomie-Status, nicht nach Unabhängigkeit. Es darf bezweifelt werden, ob diese Ausführungen zum heutigen Zeitpunkt noch korrekt sind. Der Kampf der Untergrundarmee führte dazu, daß man im Westen die Albaner nicht mehr nur als Opfer ansieht; vielleicht hätte das beherzte Eingreifen der UNO, der NATO oder der OSZE die aktuelle Eskalation der Gewalt auf beiden Seiten verhindern können? Nach der Lektüre dieses kleinen, aber ausgezeichnet formulierten und mit hilfreichen Karten ausgestatteten Bandes, der Pflichtlektüre für alle Entscheidungsträger sein sollte, versteht man die Gründe für die jetzige Situation auf dem Amselfeld. Das Buch schließt mit einer Reihe von Photos brutal mißhandelter Albaner, die einen deprimierenden Kontrast zu den 1997 geäußerten eindringlichen Worten Donika Gervallas (S. 79) bilden: "Die Kosova-Albaner versuchen nicht nur, sich von Serbien zu trennen, sondern auch ihr Land Stück für Stück zu demokratisieren. Einmal soll ein demokratischer Anfang auf dem Balkan gemacht werden! Ob dies gelingt, hängt von der internationalen Unterstützung der Kosova-Albaner ab."

Dagmar Reimmann

Erika Schläppi

Menschenrechte in der bilateralen Entwicklungszusammenarbeit

Chancen und Grenzen aus völkerrechtlicher Sicht

Nomos-Verlag, Baden-Baden, 1998, 392 S., DM 98,--

Der hohe Stellenwert, den der Schutz der Menschenrechte im Rahmen der Entwicklungszusammenarbeit genießt, ist heute unbestritten. Kaum ein internationales Dokument, das in 
den vergangenen Jahren zu Fragen der Kooperation zwischen Industrie- und Entwicklungsländern verabschiedet wurde, läßt die menschenrechtliche Dimension unerwähnt. Daß Fortschritte auf dem Gebiet wirtschaftlicher Entwicklung in einem engen Konnex zur Gewährleistung der grundlegenden Menschenrechte stehen, ist nicht nur von der Wiener UNO-Menschenrechtskonferenz im Jahr 1993 thematisiert worden, sondern in Teilaspekten auch Gegenstand der übrigen Weltkonferenzen gewesen, die die Vereinten Nationen seit Beginn dieser Dekade zu globalen Problemen veranstaltet haben. Völkerrechtliche Abkommen, die als Grundlage der Zusammenarbeit zwischen Industrie- und Entwicklungsländern dienen, enthalten heute vielfach ein eindeutiges Bekenntnis zu den Menschenrechten; im Maastrichter Unions vertrag von 1992 wird die Förderung der Menschenrechte ausdrücklich als eine der Zielsetzungen der gemeinschaftlichen Entwicklungspolitik hervorgehoben. Anliegen der hier vorzustellenden Dissertation von Erika Schläppi ist die systematische Aufarbeitung und Darstellung der völkerrechtlichen Fragen, die sich aus der Integration des Menschenrechtsschutzes in die bilaterale staatliche Entwicklungszusammenarbeit ergeben. Die Untersuchung ist bewußt auf die konzeptionellen Grundlagen dieses Themenkomplexes beschränkt, da eine Auseinandersetzung mit der Bedeutung einzelner Menschenrechte im Entwicklungsprozeß den Rahmen der Arbeit - wie die Autorin in der Einleitung wohl zu Recht feststellt - bei weitem gesprengt hätte.

Schläppi beginnt ihre Untersuchung mit einer Darstellung der Entwicklung menschenrechtlicher Standards auf der Völkerrechtsebene. Die einzelnen Rechtsquellen des internationalen Menschenrechtsschutzes, die Unterteilung der Menschenrechte in verschiedene "Generationen" und die Qualifizierung der völkerrechtlich anerkannten Menschenrechte als Verpflichtungen erga omnes sowie als internationales ius cogens stehen im Zentrum dieses einleitenden Teils der Arbeit. Ausführlich befaßt sich Schläppi zudem mit der Frage nach dem universellen Geltungsanspruch der Menschenrechte. Der Universalitätsgrundsatz könne nach dessen ausdrücklicher Bestätigung durch die Wiener Menschenrechtskonferenz aus der Perspektive des Völkerrechts und der internationalen Politik nicht mehr in Zweifel gezogen werden, allerdings müsse bei der praktischen Umsetzung der universell geltenden Menschenrechte der lokale kulturelle Kontext ausreichende Beachtung finden. Um einer Instrumentalisierung universalistischer bzw. relativistischer Positionen zu politischen Zwecken, die mit menschenrechtspolitischen Anliegen nur wenig zu tun haben, entgegenwirken zu können, ist nach Ansicht der Autorin der effiziente Einsatz unabhängiger multilateraler Kontrollmechanismen zur Durchsetzung der Menschenrechte zu empfehlen. Wenn Repräsentanten möglichst vieler verschiedener Weltregionen in den jeweiligen Kontrollgremien vertreten sind - wie dies z.B. bei der UNO-Menschenrechtskommission der Fall ist -, dann sei auch gewährleistet, daß die einzelstaatliche Umsetzung der universell geltenden Menschenrechte mit der nötigen kulturellen Sensibilität überwacht werde.

In den folgenden Abschnitten der Arbeit erörtert Schläppi die unterschiedlichen Handlungsspielräume der Staaten bei der Erfüllung ihrer Pflichten auf dem Gebiet des internationalen Menschenrechtsschutzes sowie die bereits bestehenden multilateralen Durchsetzungsmechanismen. In ihrer Untersuchung bezieht sie dabei auch die Arbeit der nicht- 
gouvernementalen Organisationen mit ein, deren Bedeutung bei der Umsetzung internationaler Menschenrechtsstandards vor allem in der Informationsbeschaffung und der Mobilisierung der öffentlichen Meinung liegt. Gleichfalls aufgezeigt werden die verschiedenen Möglichkeiten der Staaten, auf bilateraler Ebene sich für die Beachtung der Menschenrechte einzusetzen. Die Gründe für das auch nach fünfzig Jahren universeller Menschenrechtspolitik noch immer zu konstatierende Durchsetzungsdefizit erblickt Schläppi u.a. darin, daß menschenrechtlichen Anliegen in außenpolitischen Zielkonflikten nur selten eine prioritäre Stellung eingeräumt wird. Die ideologisch-geopolitische Instrumentalisierung der Menschenrechte, praktische Schwierigkeiten bei der Feststellung und Bewertung von Menschenrechtsverletzungen, die bislang eher geringe Bedeutung präventiver Elemente in der Menschenrechtspolitik und die in manchen Ländern zu beobachtenden Probleme bei der Aufrechterhaltung des staatlichen Gewaltmonopols stellen nach Ansicht der Autorin weitere Ursachen für die Diskrepanz zwischen Anspruch und Realität im internationalen Menschenrechtsschutz dar.

In ihren weiteren Ausführungen zeichnet Schläppi zunächst die während der vergangenen Jahrzehnte unternommenen Bemühungen um eine Integration menschenrechtspolitisch und entwicklungspolitisch orientierter Denkansätze auf der Ebene internationaler Organisationen und in der bilateralen Entwicklungszusammenarbeit nach, bevor sie sich im Anschluß daran mit den konzeptionellen Verbindungen zwischen den beiden Politikfeldern auseinandersetzt. Zentrale Aspekte dieses Abschnitts der Untersuchung bilden die völkerrechtlichen Verpflichtungen von Entwicklungshilfegebern im Bereich des Menschenrechtsschutzes und das damit verbundene Problem der Konditionalisierung der Hilfe, darüber hinaus aber auch die in der politikwissenschaftlichen Literatur bereits seit längerem intensiv diskutierte Frage, inwieweit sich wirtschaftliche Entwicklung einerseits und Demokratie und Achtung der Menschenrechte andererseits gegenseitig bedingen. Deutlich wendet sich die Autorin gegen die These, daß ein niedriger Entwicklungsstand Menschenrechtsverletzungen rechtfertigen könne; zu Recht weist sie auch darauf hin, daß eine solche Argumentation jeglicher völkerrechtlichen Grundlage entbehrt. Nach Ansicht Schläppis setzt nachhaltige Entwicklung einen gewissen menschenrechtlichen Mindeststandard voraus, zu dem zentrale Aspekte persönlicher Freiheit und Sicherheit, individueller Bewegungsfreiheit und des Diskriminierungsgebotes zuzurechnen sind. Wo derartige Rahmenbedingungen nicht zu verzeichnen seien, könnten auch keine nachhaltigen Entwicklungsfortschritte erzielt werden.

Den völkerrechtlichen Bedingungen und Grenzen einer aktiven Menschenrechtspolitik im Rahmen der Entwicklungszusammenarbeit ist der abschließende Teil der Untersuchung gewidmet. Zu den Kriterien, die die Autorin in ihre Analyse mit einbezieht, zählen u.a. das Einmischungsverbot und die internen Dimensionen des Selbstbestimmungsrechts, das völkerrechtliche Gewaltverbot, das Prinzip der Staatengleichheit, die Grundsätze der internationalen Zusammenarbeit und Solidarität sowie die verschiedenen Ausprägungen des völkerrechtlichen Selbsthilferechts. Auch die völkervertragsrechtlichen Aspekte des Themas - insbesondere die Frage, inwieweit sich aus der Nichterfüllung von Vertragspflichten 
oder aufgrund einer veränderten Menschenrechtssituation im Empfängerland ein Recht zur Vertragsauflösung für die Geberstaaten ergibt - werden in diesem Abschnitt ausführlich erörtert. Insgesamt enthält die Dissertation einen sorgfältig gearbeiteten und präzisen Überblick über alle völkerrechtlich relevanten Problemfelder des Beziehungsgefüges "Menschenrechte und Entwicklungszusammenarbeit". Wer sich über die Entwicklung und den heutigen Stand der Diskussion zu den verschiedenen Teilkomplexen dieser Thematik umfassend informieren will, wird die Arbeit von Schläppi sicherlich mit großem Gewinn lesen.

Markus Kaltenborn

\section{Imke Sommer}

\section{Zivile Rechte für Antigone}

$\mathrm{Zu}$ den rechtstheoretischen Implikationen der Theorie von Luce Irigaray

Schriften zur Gleichstellung der Frau, Bd. 18

Nomos-Verlagsgesellschaft, Baden-Baden, 1998, 198 S., DM 59,--

Der Fall der Antigone beschäftigt die Rechtsphilosophie seit über zwei Jahrtausenden ${ }^{1}$. Er gilt vor allem als klassisches Beispiel eines Konflikts zwischen gesetztem Recht und Naturrecht: Gestützt auf das göttliche Gebot, den toten Bruder zu bestatten, widersetzt sich Antigone ihrem Onkel Kreon, der als Herrscher die Bestattung des von ihm zum Landesverräter Erklärten verbietet. Dabei ist das Verhältnis von Mann und Frau kein zufälliges. Während das weltliche Gesetz des Mannes der Staatsräson dient, wird der Frau das göttliche, sittliche Gebot zum Dienst am Verwandten und namentlich zur Sorge um den Toten zugeschrieben. Diesen Gesichtspunkt, der sich bei Sophokles andeutet, hat vor allem Hegel in den Mittelpunkt gerückt. Sommer kommt, geleitet von den theoretischen Aussagen der französischen Neostrukturalistin Luce Irigaray, zu einer anderen Deutung: Nicht nur das Gesetz Kreons sei männliches Recht, sondern auch das ihm widersprechende göttliche Gebot. Antigone stehe zwischen zwei ihr gleichermaßen fremden Normen, an denen sie zugrunde gehe. Eine Lösung zeichne sich erst ab, wenn sie die beiden Gebote in Frage stelle und nach anderen, dritten Wegen suche. Eine Rechtsordnung, die Frauen zu ihrem Recht kommen lassen wolle, müsse also Rechte gewähren, die ihnen gestatten, solche Wege zu finden und zu gehen.

Dies ist, verkürzt, der Gedankengang, den Sommer in zwei Hauptteilen und insgesamt sieben Kapiteln darlegt. Bevor sie im zweiten Teil die eigentliche Rechtstheorie entfaltet, wird im ersten, "Grundlagen" überschriebenen Teil der Neostrukturalismus namentlich in

Siehe nur H. Hofmann, Neuere Entwicklungen in der Rechtsphilosophie, 1996, S. 5 ff.; zu unterschiedlichen Deutungen auch E. Schmidhäuser, Verbrechen und Strafe, 1995, S. 85 ff. 